\title{
Explaining the Statistical Properties of Fast Radio Bursts with Suppressed Low-frequency Emission
}

\author{
Vikram Ravi ${ }^{1,2}$ (1) and Abraham Loeb ${ }^{1}$ (1) \\ ${ }^{1}$ Harvard-Smithsonian Center for Astrophysics, 60 Garden Street, Cambridge, MA 02138, USA; vikram.ravi@cfa.harvard.edu \\ ${ }^{2}$ Cahill Center for Astronomy and Astrophysics, MC 249-17, California Institute of Technology, Pasadena, CA 91125, USA \\ Received 2018 October 31; revised 2019 February 10; accepted 2019 February 13; published 2019 March 26
}

\begin{abstract}
The possibility of fast radio burst (FRB) emission being suppressed at low frequencies, resulting in a cutoff of the average rest-frame spectrum, has been raised as an explanation for the lack of detections at meter wavelengths. We examine propagation effects that could cause this suppression, and find that a low-frequency spectral cutoff may be generic regardless of the specific FRB emission mechanism. We then illustrate the effects of a low-frequency spectral cutoff on the statistics of FRBs, given a cosmological source population. The observed FRB rate peaks at a specific frequency under a variety of assumptions. Observations at lower frequencies are more sensitive to highredshift events than observations above the maximal-rate frequency, and therefore result in more sharply broken fluence distributions. Our results suggest that the absence of low-frequency FRBs, and the differences between the Parkes and the Australian Square Kilometre Array FRB samples, can be fully explained by suppressed lowfrequency FRB emission.
\end{abstract}

Key words: methods: statistical - opacity - plasmas - radiative transfer - radio continuum: general

\section{Introduction}

In the past year, the range of frequencies over which fast radio bursts (FRBs) have been detected has been extended up to $8 \mathrm{GHz}$ (Gajjar et al. 2018), and down to $400 \mathrm{MHz}$ (CHIME/FRB Collaboration et al. 2019). FRB detection rates at frequencies between $400 \mathrm{MHz}$ and $1.8 \mathrm{GHz}$ are poised to improve by orders of magnitude with the recent advent of searches with the Canadian H I Intensity Mapping Experiment (CHIME; The CHIME/FRB Collaboration et al. 2018), the Australian SKA Pathfinder (ASKAP; Shannon et al. 2018), and the Deep Synoptic Array (V. Ravi et al. 2019, in preparation). It is therefore timely to assess the physical processes that shape FRB spectra, and their implications for FRB observations at different frequencies.

Individual FRBs have entirely disparate spectra even within the typical observation bandwidths of a few hundred $\mathrm{MHz}$ around $1.4 \mathrm{GHz}$ (Law et al. 2017; Macquart et al. 2019; Ravi 2019). Spectral structures on scales of $\sim 100 \mathrm{kHz}$ to tens of $\mathrm{MHz}$ are sometimes present even in the same FRB (Ravi et al. 2016). However, the spectra of individual events may be shaped by stochastic processes, such as the intrinsic emission mechanism as in single pulses from pulsars (Kramer et al. 2003), and time-variable diffractive scintillation effects (Cordes et al. 2017). Our focus here is instead on the astrophysics of the characteristic spectrum of the FRB phenomenon, averaged over a large ensemble of events. As we shall show (see also Vedantham et al. 2016; Fialkov \& Loeb 2017; Macquart \& Ekers 2018b), the characteristic FRB spectrum is a critical determinant of the observed population demographics.

As they involve coherent radio emission, FRBs are expected to be characterized by decreasing power-law spectra (indices $\alpha<0$ ) in the upper sections of their observed bandwidths. ${ }^{3}$ This is the case for both normal and giant ${ }^{4}$ pulses from pulsars (Kramer et al. 2003; Mikami et al. 2016), which are most

\footnotetext{
We define the spectral index, $\alpha$, using a power-law flux density or fluence spectrum $\propto \nu^{\alpha}$.

4 Meyers et al. (2017) recently presented evidence for low-frequency flattening of the spectra of Crab giant pulses.
}

nearly analogous to FRBs among observed astronomical phenomena. This is also expected from models for the FRB emission mechanism (Kumar et al. 2017, and references therein), which generally predict emission from coherent patches of particles with power-law energy distributions. However, pulsar observations (Kijak et al. 2011; Bilous et al. 2016; Murphy et al. 2017; Jankowski et al. 2018) and predictions for the environments of FRB progenitors (Kulkarni et al. 2015; Yang et al. 2016) combine to support the possibility of a spectral peak for FRBs, below which the characteristic FRB spectrum also decreases.

The lack of FRB detections prior to the recent CHIME events at frequencies $\nu_{\mathrm{obs}}<700 \mathrm{MHz}$ has led to the common belief that the FRB rate at these low frequencies is exceedingly low. ${ }^{5}$ A stacking analysis of 23 bandpass-calibrated FRB detections from ASKAP suggests a mean observed spectral index of $\alpha=-1.6_{-0.2}^{+0.3}$ between 1129 and $1465 \mathrm{MHz}$ (Macquart et al. 2019). Most efforts to observationally infer the characteristic FRB spectrum at lower frequencies have focused on comparing detection rates at different frequencies with the $1.4 \mathrm{GHz}$ rate set by the Parkes telescope (Karastergiou et al. 2015; Burke-Spolaor et al. 2016; Rowlinson et al. 2016; Caleb et al. 2017; Chawla et al. 2017). The nondetection in the 300-400 MHz GBT North Celestial Cap survey (Chawla et al. 2017 ) is the most constraining, because the large survey time (84 days) was complemented by a better sensitivity ( $3.15 \mathrm{Jy} \mathrm{ms}$ for a $5 \mathrm{~ms}$ FRB) than the $1.4 \mathrm{GHz}$ Parkes surveys. A characteristic spectral index of $\alpha>-0.3$ between the GBT and Parkes observing bands was derived, assuming a power-law FRB spectrum, even after accounting for pulse broadening caused by scattering in inhomogeneous plasma. A complementary approach was adopted by Sokolowski et al. (2018), who presented nondetections of seven bright ASKAP FRBs between 170 and $200 \mathrm{MHz}$ with the Murchison Widefield Array (MWA).

\footnotetext{
5 This possibility will be better investigated in future CHIME searches, as commissioning of the instrument is completed (CHIME/FRB Collaboration et al. 2019).
} 
This result suggests a spectral index between the MWA and ASKAP bands that is shallower than $\alpha \sim-1$.

Here we evaluate the astrophysical and observational implications of a peak or turnover frequency, $\nu_{\text {peak }}$, in the characteristic rest-frame FRB spectrum. In Section 2, we first show that several physical mechanisms can lead to the existence of a low-frequency cutoff for FRBs. An observational constraint on $\nu_{\text {peak }}$ can in turn be used to derive physical parameters of FRB progenitors and their environments. Second, besides shaping the frequencydependent FRB rate, the presence of a low-frequency spectral cutoff modifies the fluence and redshift distributions of FRBs observed at different frequencies. We demonstrate these effects in Section 3. We discuss the observational consistencies and predictions of a low-frequency cutoff for FRBs in Section 4, and conclude in Section 5. In particular, we assert that the form of the characteristic FRB spectrum can independently explain other important observed features of the FRB population, such as the differences between the Parkes and ASKAP FRB samples (James et al. 2018; Shannon et al. 2018). Throughout our discussion, we adopt the latest Planck cosmological parameters (Planck Collaboration et al. 2016), with $H_{0}=67.7 \mathrm{~km} \mathrm{~s}^{-1} \mathrm{Mpc}^{-1}$, $\Omega_{b}=0.0486, \Omega_{M}=0.3089$, and $\Omega_{\Lambda}=0.6911$.

\section{Low-frequency Modifications to FRBs}

\subsection{Physical Mechanisms}

We consider propagation effects that can suppress the observed emission from FRBs at frequencies $\nu<\nu_{\text {peak }}$. Our analysis makes no assumptions about the intrinsic FRB emission mechanism. We make a distinction between the effects we consider and those that decrease the observed signalto-noise ratio while preserving fluence, such as temporal broadening due to stochastic multipath propagation through an inhomogeneous plasma. This is important because the fluence completeness thresholds of surveys can be well defined (e.g., Keane \& Petroff 2015) and controlled for in comparing observations at different frequencies. We also do not consider the mechanisms that shape the spectra of individual FRBs, because of the likely possibility that single bursts are realizations of a stochastic process with underlying stable ensemble characteristics.

\subsubsection{Plasma Absorption}

Electromagnetic radiation cannot propagate through a plasma at frequencies

$$
\nu \lesssim \nu_{p}=\left(\frac{n_{e} e^{2}}{\pi m_{e}}\right)^{1 / 2} \approx 90\left(\frac{n_{e}}{10^{8} \mathrm{~cm}^{-3}}\right)^{1 / 2} \mathrm{MHz}
$$

where $n_{e}$ is the electron density, $e$ is the electron charge, and $m_{e}$ is the electron mass. The plasma period, $\nu_{p}^{-1}$, corresponds to the characteristic timescale of Langmuir oscillations, or relaxations of density fluctuations in a plasma. If the electron temperature, $T_{e}$, is significant, such that the plasma is relativistic (i.e., $\sqrt{k_{\mathrm{B}} T_{e} / m_{e}} \sim c$, where $k_{\mathrm{B}}$ is Boltzmann's constant, corresponding to $T_{e} \gtrsim 10^{9} \mathrm{~K}$ ), the plasma frequency is modified (Akhiezer et al. 1975).

\subsubsection{Razin-Tsytovich Effect}

The Razin-Tsytovich (e.g., Ginzburg \& Syrovatskii 1965) effect was first considered in terms of synchrotron emission from relativistic electrons within a thermal plasma. The effect of the refractive index in an electron plasma being less than unity is to widen the cone of relativistic beaming of the emission from individual electrons. This occurs for frequencies

$$
\nu \lesssim \nu_{R}=\gamma \nu_{p} \approx 2.9\left(\frac{n_{e}}{10^{8} \mathrm{~cm}^{-3}}\right)\left(\frac{B}{1 \mathrm{G}}\right)^{-1} \mathrm{GHz},
$$

where $B$ is the magnetic field strength in the emission region, and $\gamma$ is the electron Lorentz factor, which we eliminate by setting the emission frequency to $\gamma^{2}$ times the cyclotron gyrofrequency. We can express $B$ in terms of $n_{e}$ and $T_{e}$ for a thermal plasma assuming equipartition to find $\nu_{R} \approx 400\left(n_{e} / \mathrm{cm}^{-3}\right)^{1 / 2}\left(T_{e} / \mathrm{K}\right)^{-1 / 2} \mathrm{MHz}$. This likely provides a lower limit on the characteristic frequency. This suppression was found to be applicable to relativistic bremsstrahlung emission by Melrose (1972), and to coherent emission in pulsar magnetospheres by Arons \& Barnard (1986). As above, Equation (2) applies only to a nonrelativistic plasma. The Razin-Tsytovich effect has possibly been observed in solar radio bursts (Boischot \& Clavelier 1967).

\subsubsection{Stimulated Raman Scattering}

In the case of sources with high brightness temperatures, and therefore high emanent radiation energy densities, radio emission can also be Raman-scattered by Langmuir waves in dense plasma (Gangadhara \& Krishan 1992; Levinson \& Blandford 1995). Although the scattering minimally affects the spectra of isotropically radiating sources, sources that are beamed toward the observer are affected by the scattering of radiation away from the line of sight. The growth of the Langmuir oscillations in response to incident radiation is nonlinear in the radiation energy density, and therefore in the brightness temperature $T_{b}$. Adopting a fiducial FRB brightness temperature of $10^{36} \mathrm{~K}$ (Katz 2014), ${ }^{6}$ strong stimulated Raman scattering (Levinson \& Blandford 1995) is in effect for

$$
\begin{gathered}
\nu \lesssim 130\left(\frac{n_{e}}{\mathrm{~cm}^{-3}}\right)^{1 / 2}\left(\frac{T_{e}}{\mathrm{~K}}\right)^{-1 / 2} \mathrm{MHz} \\
\frac{\nu}{4.5 \times 10^{-13} \mathrm{MHz}} \gtrsim\left(\frac{n_{e}}{\mathrm{~cm}^{-3}}\right)^{1 / 2}\left(\frac{T_{b}}{10^{36} \mathrm{~K}}\right)^{-1}\left(\frac{T_{e}}{\mathrm{~K}}\right)^{-3 / 2} .
\end{gathered}
$$

The "weak" case of stimulated Raman scattering identified by Levinson \& Blandford (1995) only modifies the latter of these two conditions. Hence, Equation (3) is a strong constraint on the electron density and temperature of the medium surrounding FRB sources.

\subsubsection{Induced Compton Scattering}

In contrast to the case of stimulated Raman scattering, induced Compton scattering results in radio photons losing significant energy to thermal electrons in the presence of sufficient photon and electron densities (e.g., Coppi et al. 1993). These requirements are satisfied for a Thomson optical depth in excess of $0.02\left[T_{b} /\left(10^{12} \mathrm{~K}\right)\right]^{-1}$. For an assumed FRB

\footnotetext{
6 An FRB at a redshift of $z=0.5$ with a mean flux density of $1 \mathrm{Jy}$, and a duration equal to the source light-crossing time of $1 \mathrm{~ms}(1 / 1.5 \mathrm{~ms}$ in the rest frame), corresponds to $T_{b} \approx 10^{36} \mathrm{~K}$.
} 
brightness temperature of $T_{b} \approx 10^{36} \mathrm{~K}$ and a source radius $r_{\text {src }}=3 \times 10^{7} \mathrm{~cm}$, the requirement of a Thomson optical depth below the aforementioned value places an upper bound on the electron density of

$$
n_{e} \lesssim 10^{-9}\left(\frac{r_{\mathrm{src}}}{3 \times 10^{7} \mathrm{~cm}}\right) \mathrm{cm}^{-3}
$$

This is an extremely strong constraint, and possibly conservative given that FRB emission is likely beamed. We note that in the regime where both stimulated Raman and induced Compton scattering are active, Raman scattering likely dominates (Levinson \& Blandford 1995).

One might infer that the high brightness temperature of pulsar emission implies that induced Compton scattering is important in that scenario as well. This has been shown not to be the case (e.g., Wilson \& Rees 1978), because of the relativistic nature of pulsar winds. Thermal plasma in supernova remnants surrounding young pulsars is also not significant. Consider the highest brightness temperature reported for a $\mathrm{Crab}$ giant pulse of $10^{41} \mathrm{~K}$ for an estimated emission region size of $10 \mathrm{~cm}$ (Hankins \& Eilek 2007). For $r_{\text {src }} \sim 10^{16} \mathrm{~cm}$ in the inner regions of supernova remnants, we require $n_{e} \gtrsim 3 \times 10^{7} \mathrm{~cm}^{-3}$, in contrast to the requirement of $n_{e} \gtrsim 0.3 \mathrm{~cm}^{-3}$ for FRBs.

The spectral distortions caused by induced Compton scattering mildly suppress the spectral energy distributions of sources at all frequencies where the brightness temperature is sufficiently high. Although the specific distortions are dependent on the geometry of the source (Coppi et al. 1993), induced Compton scattering for a spherical source permeated by or embedded within a spherical cloud of thermal plasma will have a spectral index of $\alpha=1$ for a flat or positively sloped input spectrum, and $\alpha=1-\alpha^{\prime} / 2$ for an input spectrum with an input spectral index of $-\alpha^{\prime}$ (Sunyaev 1971). For a nonthermal source, the brightness temperature and therefore the susceptibility to induced Compton scattering varies with the emission frequency. As the actual brightness temperatures of FRBs are highly uncertain, we simply adopt the estimate of $T_{b} \approx 10^{36} \mathrm{~K}$ at emission frequencies around $1 \mathrm{GHz}$.

The above estimates of the regimes in which stimulated Raman and induced Compton scattering affect FRB propagation do not include the effects of the short pulse duration (Lyubarsky 2008; Lyubarsky \& Ostrovska 2016). In the case where the pulse is narrowly beamed, as is expected outside FRB sources, the stimulated/induced scattering rate has a growth timescale that is comparable to the pulse timescale. Therefore, the optical depths to these scattering mechanisms can depend primarily on the pulse duration, and not on the scale of the scattering medium. In general, Lyubarsky (2008) found that optical depths of order 10 are required to suppress the propagation of an individual millisecond-duration pulse in a medium far from its source. However, because the effect of pulse duration depends on the geometrical configuration of the emission region, and we are mainly interested in demonstrating the possible effects of induced scattering in the immediate vicinities of FRB sources, we do not account for the increased optical-depth requirement in the forthcoming discussion.

\subsubsection{Free-Free Absorption}

A thermal plasma surrounding an FRB source will also result in free-free absorption of the incident radiation. This effect has been previously considered as a necessary constraint to overcome in progenitor models involving young neutron stars (e.g., Kulkarni et al. 2015). Rajwade \& Lorimer (2017) considered a selection of specific models for the environments of FRB progenitors to predict the resulting FRB detection rates for upcoming sub-1 GHz telescopes such as CHIME, the Hydrogen Intensity and Real-time Analysis eXperiment (Newburgh et al. 2016), and the UTMOST experiment (Bailes et al. 2017). Defining a characteristic frequency as that below which the free-free optical depth is greater than unity, free-free absorption results in attenuation for frequencies

$$
\nu \lesssim 300\left(T_{e} / \mathrm{K}\right)^{-0.64} \mathrm{EM}^{0.48} \mathrm{MHz}
$$

where the emission measure (EM) of the absorbing plasma is in standard units of $\mathrm{pc} \mathrm{cm}^{-6}$ (Draine 2011).

\subsubsection{Propagation through Inhomogeneous Plasma}

Temporal broadening of FRBs due to multipath propagation through inhomogeneous plasma results in the emitted FRB temporal profiles being convolved with a one-sided unit-area exponential profile with an $e^{-1}$ timescale, $\tau_{d}$, that is proportional to $\nu^{\psi}$ with $\psi \sim-4$ (e.g., Ravi 2019). This effect therefore conserves fluence, and does not impact surveys with known fluence completeness thresholds. However, FRB surveys typically do not search for events that last longer than some fixed temporal width, and therefore will not detect FRBs that have been broadened beyond this maximum width. This effect is difficult to include in assessments of fluence completeness (Keane \& Petroff 2015), and may hence result in a suppression of the low-frequency FRB detection rate above a given fluence threshold, relative to higher frequencies. We consider the possible implications of this effect below in Section 3. Following the broader goal of this work, we specifically incorporate the scenario of temporal broadening caused by the immediate environments of FRB sources (e.g., CHIME/FRB Collaboration et al. 2019). We do not consider other causes of temporal broadening, for example, in the Milky Way (e.g., Burke-Spolaor \& Bannister 2014) or the circumgalactic medium (Vedantham \& Phinney 2019).

It is additionally possible that FRBs are strongly magnified by au-scale plasma lenses in their host galaxies (Cordes et al. 2017), or by the effects of constructive interference of rays propagating along multiple paths (e.g., Ravi et al. 2016). Although lensing caustics impose a rich frequency structure on the magnified input spectrum, with spectral peaks with $\sim 0.1-1 \mathrm{GHz}$ widths magnified by up to factors of $\sim 100$, the effects can be quite broadband despite the chromaticity of the plasma refractive index. Interference maxima resulting from strong scattering in FRB host galaxies, with characteristic ray delays $\tau_{d}$ producing spectral peaks with widths $\sim 1 /\left(2 \pi \tau_{d}\right)$ and an exponential intensity distribution, are also difficult to relate to low-frequency FRB suppression, because of the strong reduction of $\tau_{d}$ with redshift (stronger than $(1+z)^{3}$; Macquart \& Koay 2013). However, as noted by Macquart et al. (2019), a combination of angular broadening and interference due to scattering in both FRB host galaxies and intervening systems may conspire to magnify FRBs only at $\mathrm{GHz}$ frequencies. As the 
theory underlying these effects is only beginning to be developed, we defer consideration of the resulting impacts on FRB surveys to future work.

\subsubsection{Lessons from Galactic Pulsars}

Pulsar emission is the closest known analog to FRBs. Pulsars often have peaks or breaks at frequencies $\nu_{\text {peak }}$ between 0.1 and few GHz (Kijak et al. 2011; Bilous et al. 2016; Jankowski et al. 2018). Multiple mechanisms likely define these critical frequencies. For example, although a positive correlation may exist between $\nu_{\text {peak }}$ and DM, and some GHz-peaked spectrum pulsars are viewed along particularly dense sightlines, free-free absorption effects are difficult to disentangle from increased scattering at low frequencies (Rajwade et al. 2016, and references therein). In addition, young pulsars tend to have flatter radio spectra, and higher values of $\nu_{\text {peak }}$, than older pulsars (e.g., Jankowski et al. 2018). Although this may suggest that pulsar spectra depend sensitively on the magnetospheric properties, this trend may also be a consequence of young pulsars residing closer to their extreme birth environments, or simply a selection effect. Third, as persuasively argued by Jankowski et al. (2018), our knowledge of pulsar spectra generally improves upon closer inspection; the best studied pulsars, observed over the largest number of epochs to mitigate the effects of scattering and possible intrinsic variations, have complex broadband spectra that cannot be characterized by single power laws. As a minimum, the example of Galactic pulsars suggests that inferences on FRB spectra from direct observations (e.g., Macquart et al. 2019) will require a careful analysis of selection effects and observational incompleteness.

\subsection{Application to FRB Progenitor Environments}

Some of the physical mechanisms identified above can plausibly result in a characteristic low-frequency spectral cutoff for FRBs. Additionally, if the frequency of such a cutoff can be determined, the properties of the immediate progenitor environments of FRBs can be constrained. Figure 1 illustrates necessary values of the density and temperature of a thermal, nonrelativistic plasma surrounding (and permeating, in the case of RazinTsytovich suppression) FRB sources, for a characteristic frequency of $\nu_{\text {peak }}=500 \mathrm{MHz}$. The Razin-Tsytovich effect (Equation (2)) is evaluated assuming equipartition between the thermal and magnetic energy densities in the plasma, and the EM for the free-free absorption constraint (Equation (6)) is evaluated for a constant-density nebula with a $0.1 \mathrm{pc}$ radius.

The required combinations of $T_{e}$ and $n_{e}$ for $\nu_{\text {peak }} \leqslant$ $500 \mathrm{MHz}$ are plausible for Razin-Tsytovich suppression and stimulated Raman scattering, and for a $\sim 0.1$ pc ionized nebula in the case of free-free absorption. In practice, however, freefree absorption may not be the preferred low-frequency suppression mechanism because of the requirement for large DM contributions from the host environment. Absorption below the plasma frequency is less likely. Induced Compton scattering will occur for any combination of $T_{e}$ and $n_{e}$ in Figure 1 for ionized nebula sizes $r_{\text {src }} \lesssim 10$ pc. However, in comparison with the other suppression/absorption mechanisms, induced Compton scattering less strongly distorts the observed low-frequency spectrum.

The results in Figure 1 can be compared with expectations for different astrophysical environments that could host FRB

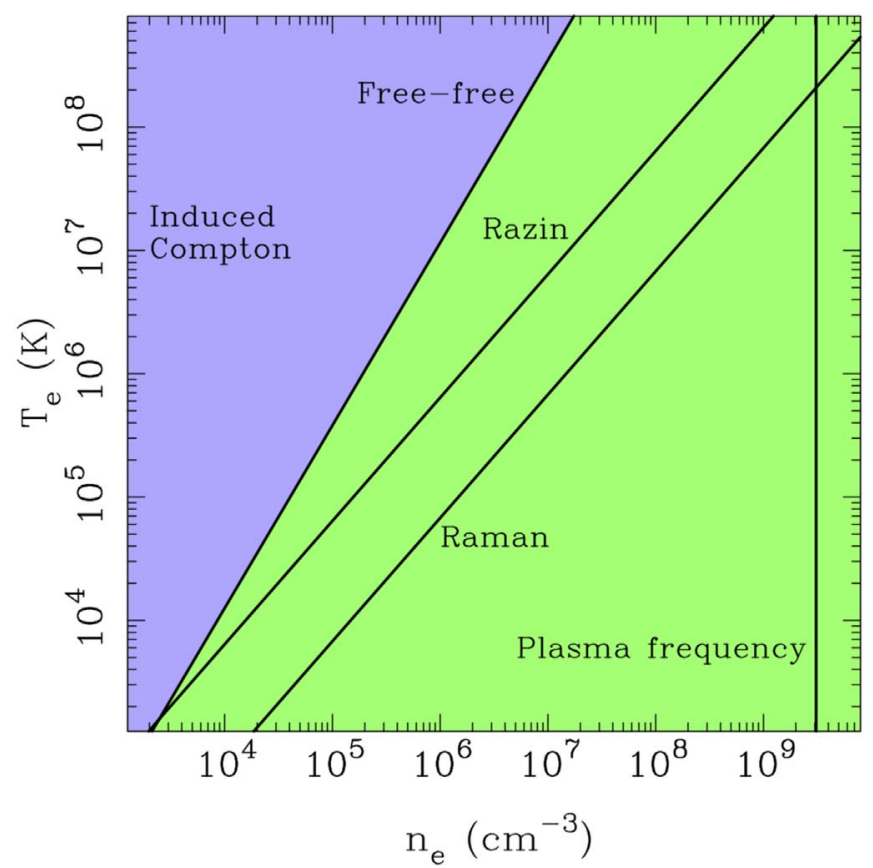

Figure 1. Loci in $T_{e}-n_{e}$ space derived from different FRB absorption/ suppression mechanisms assuming $\nu_{\text {peak }}=500 \mathrm{MHz}$. Radiation is suppressed for $\nu<500 \mathrm{MHz}$, which is generally the case for higher values of $n_{e}$ and lower values of $T_{e}$. As labeled, the lines indicate constraints from absorption below the plasma frequency (Equation (1)), Razin-Tsytovich suppression (Equation (2)) assuming a plasma beta of unity, stimulated Raman scattering (Equation (3)), and free-free absorption in a constant-density nebula of $0.1 \mathrm{pc}$ radius (Equation (6)). The green shaded region illustrates the possibly excluded region assuming that the free-free optical depth at $500 \mathrm{MHz}$ is less than unity. The purple shaded region labeled as induced Compton scattering corresponds to radiation below $\nu_{\text {peak }} \sim 1 \mathrm{GHz}$ being suppressed for ionized-nebula sizes $r_{\text {src }} \lesssim 10 \mathrm{pc}$ (Equation (5)). For example, setting $r_{\mathrm{src}}=0.1 \mathrm{pc}$ only requires a density of $n_{e}>10 \mathrm{~cm}^{-3}$ for induced Compton scattering to occur.

progenitors. FRB progenitor models generally posit a compact object as the engine, most often invoking a highly magnetized neutron star (for a summary of FRB progenitor models, see Platts et al. 2018). Young and massive compact objects are likely surrounded by dense, hot plasma. The environments of the repeating FRB (Michilli et al. 2018) and some nonrepeating events (e.g., Masui et al. 2015) are likely dense and magnetized, corresponding to young supernova remnants or the surroundings of supermassive black holes (SMBHs).

Young neutron star in its supernova remnant: Extreme emission from young pulsars (e.g., Cordes \& Wasserman 2016) or magnetars (e.g., Metzger et al. 2017) forms a leading model for FRBs. Although older supernova remnants like the Crab Nebula self-evidently do not result in absorption of radiation at $\nu \gtrsim 100 \mathrm{MHz}$, the environments of neutron stars younger than $\sim 100 \mathrm{yr}$ are more extreme. Margalit et al. (2018) computed photoionization models of superluminous supernova remnants that potentially host nascent FRB-emitting magnetars, finding $T_{e} \sim 10^{6}-10^{7} \mathrm{~K}$ in the central (post-shock) regions where number densities $n_{e} \gtrsim 10^{5} \mathrm{~cm}^{-3}$ are expected (Metzger et al. 2017). Significant pre-explosion mass loss is also inferred for some engine-driven core collapse supernovae, resulting in postshock densities of $n_{e} \gtrsim 10^{6} \mathrm{~cm}^{-3}$ within $10^{14} \mathrm{~cm}$ of the center (for a compilation, see Ho et al. 2019).

Pulsar wind: Pulsars lose most of their spindown energy to winds of relativistic particles. Assuming a bulk Lorentz 
factor of the wind of $\sim 10^{2}$ just beyond the light cylinder (Gaensler \& Slane 2006, and references therein), negligible magnetization of the wind, and a standard neutron-star radius of $10 \mathrm{~km}$, the particle density in the wind at a radius of $1000 \mathrm{~km}$ is

$$
n_{\mathrm{pw}} \sim 10^{16}\left(\frac{B_{p}}{10^{14} \mathrm{G}}\right)^{2}\left(\frac{P}{1 \mathrm{~s}}\right)^{-4},
$$

where $B_{p}$ is the polar magnetic field strength, and $P$ is the spin period. Evaluating the absorption properties of such a wind is difficult because of its relativistic nature (e.g., Wilson \& Rees 1978). The wind is also likely magnetically dominated at this location, transitioning to a particle-dominated outflow only near the termination shock, where bulk Lorentz factors of $\sim 10^{6}$ are in fact inferred.

Supermassive black holes: Wildly variable assessments exist in the literature of the plasma environments surrounding SMBHs. Alexander et al. (2016) collated inferences from radio outflows/jets from tidal disruption events to find typical densities of $10^{4}-10^{5} \mathrm{~cm}^{-3}$ at radii of $10^{15} \mathrm{~cm}$. As an example, Bondi (1952) accretion onto an $\mathrm{SMBH}$ from a $10^{6} \mathrm{~K}$ interstellar medium (ISM) will result in a density at $10^{15} \mathrm{~cm}$ that is $\sim 10^{4}\left(M_{\mathrm{BH}} / 10^{6} M_{\odot}\right)$ times higher than the ISM density, where $M_{\mathrm{BH}}$ is the SMBH mass.

In summary, a selection of plausible FRB progenitor environments can result in the suppression of emission below $\nu_{\text {peak }} \sim 1 \mathrm{GHz}$. We stress that all mechanisms, including Razin-Tsytovich suppression, stimulated Raman scattering, and induced Compton scattering, need to be accounted for in FRB progenitor models. We now turn our attention to the observational consequences of a characteristic low-frequency spectral cutoff for FRBs, addressing the consistency of this model with current observations, and predictions of this model that may allow $\nu_{\text {peak }}$ to be measured.

\section{The Observed Fluence and Redshift Distributions}

We use a straightforward fiducial model for the characteristic FRB fluence spectrum and luminosity function to demonstrate the effects of a characteristic rest-frame low-frequency spectral cutoff for FRBs. Our analysis assumes a cosmological FRB population, such that FRBs originate from redshifts wherein the extragalactic DMs are dominated by propagation through the circum- and intergalactic medium. A growing compilation of observations supports this scenario. For example, the repeating FRB 121102 is observed at a large extragalactic distance (Tendulkar et al. 2017), FRB 150807 had no nearby host galaxies within its localization region (Ravi et al. 2016), and evidence exists for a relation between fluence and DM consistent with a cosmological population (Shannon et al. 2018).

We adopt a two-component power law for the fluence spectrum:

$$
\begin{gathered}
F\left(\nu_{r}\right)=F_{0}\left(\nu_{r} / \nu_{\text {peak }}\right)^{\alpha}, \nu_{r} \geqslant \nu_{\text {peak }} \\
=F_{0}\left(\nu_{r} / \nu_{\text {peak }}\right)^{\beta}, \nu_{r}<\nu_{\text {peak }},
\end{gathered}
$$

where $\nu_{r}$ is the rest frequency, and $\alpha>0$ and $\beta<0$ are the two spectral indices. As noted above, we consider this to be a "central" FRB spectrum, which the individual rest-frame spectra of FRBs tend toward on average. Evidence for the existence of such an FRB spectrum was recently provided by
Macquart et al. (2019), who showed that the calibrated spectra of 23 ASKAP FRBs tended toward a central value upon averaging, rather than having infinite variance.

The values of $\alpha$ and $\beta$ are difficult to specify a priori. Specifically, the value of $\alpha$ is set by the intrinsic FRB emission mechanism, and $\beta$ is set by the low-frequency suppression mechanism. Induced Compton scattering will result in $\beta=1-\alpha / 2$ for $\alpha \leqslant 0$, free-free absorption will result in $\beta=2.1$, and the remaining mechanisms considered in Section 2 will result in much steeper cutoffs. For the purposes of our demonstration in this section, we assume $\alpha=-1.8$ (Macquart et al. 2019), and consider illustrative values of $\beta=2.1$ and $\beta=\infty$.

Let $n_{\text {ref }}(>F)$ be the observed number of FRBs per unit time, per comoving volume element at a fiducial redshift $z_{\text {ref }}$, a fiducial observing frequency $\nu_{\text {ref }}$, and above an observed fluence $F$. We have little guidance regarding what functional form to adopt for $n_{\text {ref }}(>F)$. For consistency with the observations of single pulses from pulsars (Mickaliger et al. 2018), but not the extreme case of giant pulse emission, we adopt a log-normal form for the differential FRB counts, $\frac{d}{d F} n_{\text {ref }}(>F)$, with mean $\ln F_{\text {ref }}$ and standard deviation $\sigma_{\text {ref }}^{2}$. Another possibility would have been the Weibull distribution, which describes the statistics of maximal extreme values. A power-law distribution has the disadvantage of having more free parameters, including the arbitrary choices of low- and high-fluence cutoffs (see Fialkov \& Loeb 2017; Macquart \& Ekers 2018b). Again for the purposes of demonstration, we adopt fiducial values of $\nu_{\text {ref }}=1 \mathrm{GHz}$, and $\sigma_{\text {ref }}=0.3 \mathrm{dex}$; we do not need to specify $F_{\text {ref. }}$. We adopt an arbitrary normalization for the total volumetric rate, and only present relative quantities.

The equivalent quantity to $n_{\text {ref }}(>F)$ at a frequency $\nu_{\text {obs }}$ and a redshift $z$ is given by

$$
n(z,>F)=R(z) \frac{\left(1+z_{\mathrm{ref}}\right)}{(1+z)} n_{\mathrm{ref}}\left(>F^{\prime}\right),
$$

where $R(z)$ captures any cosmic evolution in rest-frame FRB volumetric rate, and the effective fluence is given by

$$
F^{\prime}=F\left[\frac{D_{L}\left(z_{\mathrm{ref}}\right)}{D_{L}(z)}\right]^{-2} \frac{F\left[\left(1+z_{\mathrm{ref}}\right) \nu_{\mathrm{ref}}\right]}{F\left[(1+z) \nu_{\mathrm{obs}}\right]} \frac{\left(1+z_{\mathrm{ref}}\right)}{(1+z)} .
$$

Here, the $\left[\frac{D_{L}\left(z_{\text {ref }}\right)}{D_{L}(z)}\right]^{-2}$ factor captures the evolution of flux density with luminosity distance $D_{L}$, the $\frac{F\left[\left(1+z_{\text {ref }}\right) \nu_{\text {ref }}\right]}{F\left[(1+z) \nu_{\text {obs }}\right]}$ factor corrects the observed fluence for the redshifted frequency (which we refer to as the $K$-correction; Hogg et al. 2002), and the $\frac{\left(1+z_{\text {ref }}\right)}{(1+z)}$ factor corrects the observed fluence for the dilated duration.

The effects of temporal broadening of FRBs beyond the maximum width-detection thresholds of surveys, discussed above in Section 2.1.6, can be incorporated into Equation (10). Let a fraction $f_{b \text {,ref }}$ of FRBs observed at a frequency $\nu_{\text {ref from a }}$ redshift $z_{\text {ref }}$ be broadened beyond observation, such that they have widths $\tau_{d}>\tau_{s}$. The evolution of the broadened FRB fraction, $f_{b}$, with redshift and observing frequency depends on the form of the probability distribution function of FRBs in $\tau_{d}$, $N\left(>\tau_{d}\right)$. For the purposes of demonstrating the effects of temporal broadening, we consider a single scenario to compare with the case of $f_{b}=0$ always. In this scenario, we assume 
$N\left(>\tau_{d}\right) \propto \tau_{d}^{-1}$ for $0 \leqslant \tau_{d}<\tau_{\mathrm{m}}$, corresponding to a uniform probability-density function in $\tau_{d}$, where the upper cutoff $\tau_{\mathrm{m}}$ depends on $z$ and $\nu_{\text {obs }}$. We further assume no redshift evolution in $N\left(>\tau_{d}\right)$ for a fixed rest-frame frequency. Then, the evolution of $f_{b}$ is described by

$$
\begin{gathered}
f_{b}=1-\left(1-f_{b, \text { ref }}\right)\left[\frac{\left(1+z_{\text {ref }}\right) \nu_{\text {ref }}}{(1+z) \nu_{\mathrm{obs}}}\right]^{\psi}, \tau_{\mathrm{m}}>\tau_{s} \\
=1, \tau_{\mathrm{m}} \leqslant \tau_{s} .
\end{gathered}
$$

Given our assumptions about $N\left(>\tau_{d}\right)$, the value of $\tau_{\max }$ is specified relative to $\tau_{s}$ for a fixed $f_{b \text {,ref }}$. Equation (10) can then be modified by adding a pre-factor $\left(1-f_{b}\right)$. We assume $\psi=-4$ in this demonstration (Ravi 2019).

The number of observed FRBs above a fluence $F$, at a frequency $\nu_{\mathrm{obs}}$, is given by the redshift integral over Equation (10):

$$
N(>F)=\int_{0}^{\infty} n(z,>F) \frac{4 \pi d^{2} V_{C}}{d \Omega d z} d z
$$

where $d^{2} V_{C} /(d \Omega d z)$ is the standard comoving volume element. The redshift distribution of FRBs observed above a fluence $F$, at a frequency $\nu$, is then

$$
\frac{d}{d z} N(>F)=n(z,>F) \frac{4 \pi d^{2} V_{C}}{d \Omega d z} .
$$

\subsection{Frequency-dependent Detection Rate}

We begin by illustrating the effects of a characteristic lowfrequency spectral cutoff for FRBs by calculating the detection rates corresponding to various values of $\nu_{\text {peak }}$ for surveys at different frequencies. We assume that all surveys have identical fluence thresholds, corresponding to $F_{\text {ref }}$, and that all surveys search over the same DM ranges. This latter assumption aids in evaluating the redshift integral in Equation (14) by setting a maximum redshift. We assume a maximum DM of $6000 \mathrm{pc} \mathrm{cm}^{-3}$, which we assume originates predominantly in the circum- and intergalactic medium, and which therefore crudely corresponds to a redshift of 6 (e.g., Ioka 2003). Finally, we assume no redshift evolution of the volumetric FRB rate (constant $R(z)$ in Equation (10)). The results are shown in Figure 2.

For an FRB spectrum described by $\alpha=-1.8$ and $\beta=2.1$, the correlation between $\nu_{\text {peak }}$ and the frequency at which the detection rate peaks is evident. At high frequencies, all cases approach the same curve as all detectable sources are observed at rest frequencies above $\nu_{\text {peak }}$. The lower values of $\nu_{\text {peak }}$ result in higher detection rates at low frequencies because sources are observed above $\nu_{\text {peak }}$ at lower redshifts, where more of the population is accessible. The FRB spectrum with a sharp cutoff below $\nu_{\text {peak }}=1 \mathrm{GHz}$ has a lower detection rate at low frequencies than the former case with the same $\nu_{\text {peak }}$, because sources are no longer amplified into the detection volume by the negative $K$-correction. The effects of scattering in this latter case, in the specific scenario considered here, are to further suppress the detection rates at lower frequencies as more events are temporally broadened beyond detection.

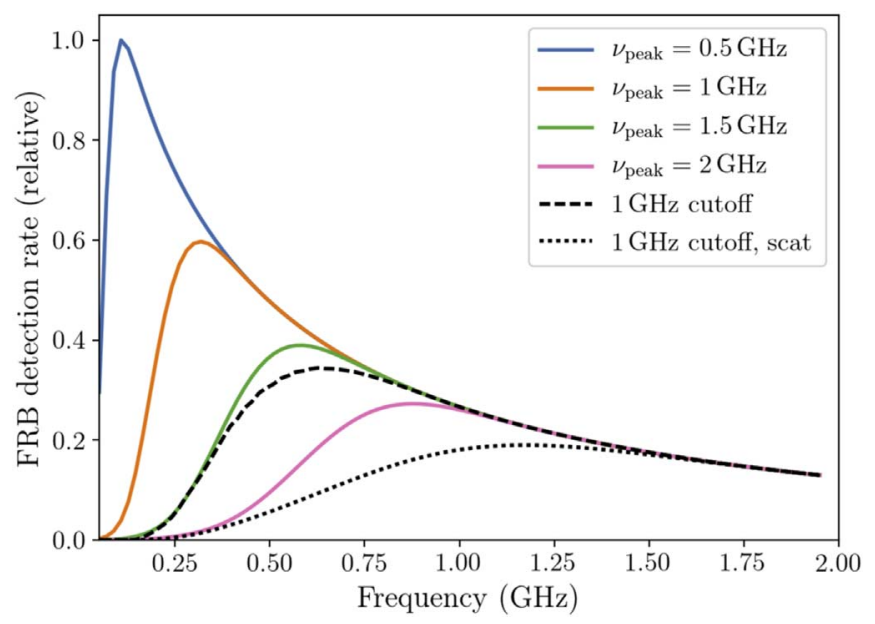

Figure 2. Relative FRB detection rates at different frequencies. We show results for an FRB spectrum described by $\alpha=-1.8$ and $\beta=2.1$ for four values of $\nu_{\text {peak }}$ (solid curves), and for an FRB spectrum with $\alpha=-1.8$ and a sharp cutoff $(\beta=\infty)$ below $\nu_{\text {peak }}=1 \mathrm{GHz}$ (dashed curve). These results all do not include the effects of temporal broadening caused by multipath propagation $\left(f_{b}=0\right)$. As a comparison, we recompute the sharp-cutoff case with $f_{b \text {,ref }}=0.1$ (dotted curve). We assume FRB detection thresholds at each frequency that are equivalent to $F_{\text {ref }}$, and set the maximum FRB redshift to 6 . We set $\nu_{\text {ref }}=1 \mathrm{GHz}$ and $\sigma_{\text {ref }}=0.3 \mathrm{dex}$ to describe the FRB luminosity function.

\subsection{Observed Redshift Distributions}

We next illustrate the redshift distributions of FRBs observed at different frequencies relative to the frequency with the peak rate. We again assume a common fluence threshold of $F_{\text {ref }}$, and a constant $R(z)$ in Equation (10). The differential redshift distribution of FRBs observed above a fluence $F$ at a frequency $\nu$ is given by Equation (15). We consider observations at frequencies below $\left(\nu_{\mathrm{obs}}=0.25 \mathrm{GHz}\right)$, around $\left(\nu_{\mathrm{obs}}=0.75 \mathrm{GHz}\right)$, and above $\left(\nu_{\mathrm{obs}}=1.25 \mathrm{GHz}\right)$ the frequencies with the peak FRB rates for $\nu_{\text {peak }}=1 \mathrm{GHz}$ and $\nu_{\text {peak }}=2 \mathrm{GHz}$. Results are shown in Figure 3 for both FRB spectral models discussed above.

Observations at low frequencies tend to be more sensitive to high-redshift FRBs than observations at higher frequencies. This is because of the negative $K$-correction, which results in more distant FRBs being brighter than expected from the $D_{L}^{2}$ law as they are observed closer to their spectral peaks. The breaks evident in the left panel of Figure 3 for the $\nu_{\mathrm{obs}}=0.25 \mathrm{GHz}$ and $\nu_{\text {peak }}=1 \mathrm{GHz}$ curve, and the $\nu_{\mathrm{obs}}=$ $0.75 \mathrm{GHz}$ and $\nu_{\text {peak }}=2 \mathrm{GHz}$ curve, correspond to the redshifts where $\nu_{\text {peak }}=(1+z) \nu_{\text {obs }}$ (higher-redshift FRBs are all observed above their spectral peaks). As shown in the right panel of Figure 3, a sharper characteristic low-frequency spectral cutoff will result in a more pronounced bias toward high-redshift FRBs for low-frequency observations, together with a potentially associated drop in overall detection rate for appropriate luminosity functions and detection thresholds. This is because FRBs only become detectable at low frequencies when the emission redshifts into view. In the right panel of Figure 3, we also show the effects of temporal broadening in the specific scenario considered here. The further suppression of the lower-frequency detection rate is again evident. For a given observing frequency, this suppression is reduced at higher redshifts, as FRBs are observed at higher, less scattered rest frequencies. Temporal broadening therefore can enhance 

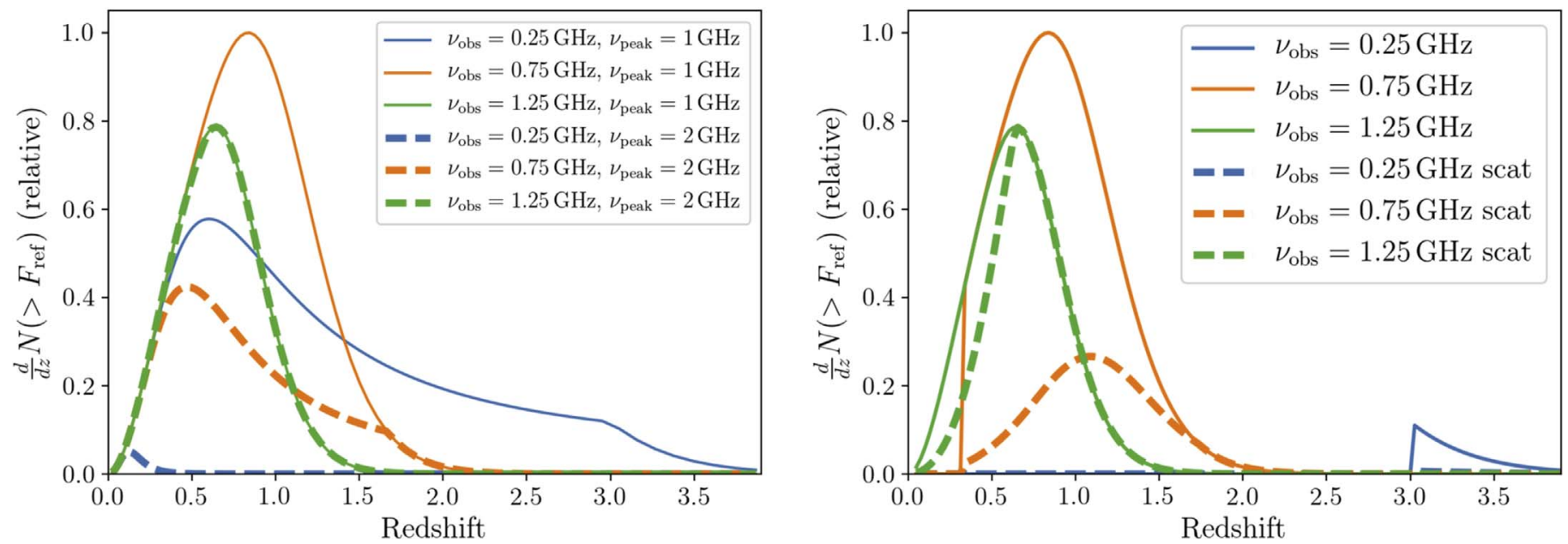

Figure 3. Left panel: the differential FRB rate at different redshifts (Equation (15)) for observations at $\nu_{\mathrm{obs}}=0.25,0.75,1.25 \mathrm{GHz}$, and $\nu_{\text {peak }}=1,2 \mathrm{GHz}$ (solid and dashed lines respectively). An FRB spectrum described by $\alpha=-1.8$ and $\beta=2.1$ is assumed, and the luminosity function is as above (e.g., Figure 2). Right panel: same as the left panel, but for an FRB spectrum with $\alpha=-1.8$ and a sharp cutoff $(\beta=\infty)$ below $\nu_{\text {peak }}=1 \mathrm{GHz}$ (solid lines). We compare this latter case with a scenario where as in Figure 2 some FRBs, quantified by $f_{b, \text { ref }}=0.1$, are temporally broadened beyond detection (dashed lines).

the tendency of low-frequency surveys to observe FRBs at higher redshifts.

\subsection{Observed Fluence Distributions}

Finally, we illustrate the fluence distributions of FRB samples observed at different frequencies relative to the frequency with the peak rate (Equation (14)). For an FRB spectrum described by $\alpha=-1.8$ and $\beta=2.1$, we calculate the fluence distributions for $\nu_{\text {peak }}=2 \mathrm{GHz}$ at $\nu_{\mathrm{obs}}=0.25,0.75$, $1.25 \mathrm{GHz}$. We also calculate the fluence distribution for the cutoff spectrum below $\nu_{\text {peak }}=1 \mathrm{GHz}$, at $\nu_{\text {obs }}=0.25 \mathrm{GHz}$. Thus, the fluence distributions we evaluate correspond exactly to the redshift distributions shown in Figure 3. The results are shown in Figure 4.

The expectation for a uniformly distributed source population in Euclidean space, which closely corresponds to a nearby cosmological source population, is a relation $N(>F) \propto F^{-3 / 2}$. At the high-fluence end of the fluence distributions for the $[\alpha=-1.8, \beta=2.1]$ spectral model in Figure 4, the curves (will) asymptote to this relation, because of the bounded nature of the assumed FRB luminosity function. The fluence distributions for values of $\nu_{\text {obs }}$ below the frequency with the peak FRB rate (which would be $\nu_{\mathrm{obs}} \approx 1 \mathrm{GHz}$; Figure 2) have a relative excess of faint events, or equivalently a paucity of bright events. Indeed, these fluence distributions are steeper than the fiducial $F^{-3 / 2}$ law in portions of their domains, approaching $F^{-2}$. This is because of the excess of higherredshift, fainter events observed at these low frequencies, indicated in Figure 3. The flatness of the fluence distribution at the higher frequency of $\nu_{\mathrm{obs}}=1.25 \mathrm{GHz}$ is due to a combination of the positive $K$-correction caused by the negatively sloped FRB spectrum observed at this frequency, and cosmic evolution of the comoving volume element.

The fluence distribution at $\nu_{\mathrm{obs}}=0.25 \mathrm{GHz}$ for the spectral model with a sharp cutoff below $\nu_{\text {peak }}=1 \mathrm{GHz}$ is quite different from the above cases. High-fluence, nearby events are no longer present because of the lack of low-redshift FRBs radiating at the observing frequency; the $F^{-3 / 2}$ behavior at high fluences is no longer evident. Low-fluence events are also suppressed by the more stringent detectability constraint on the most distant FRBs. A similar form for the fluence distribution

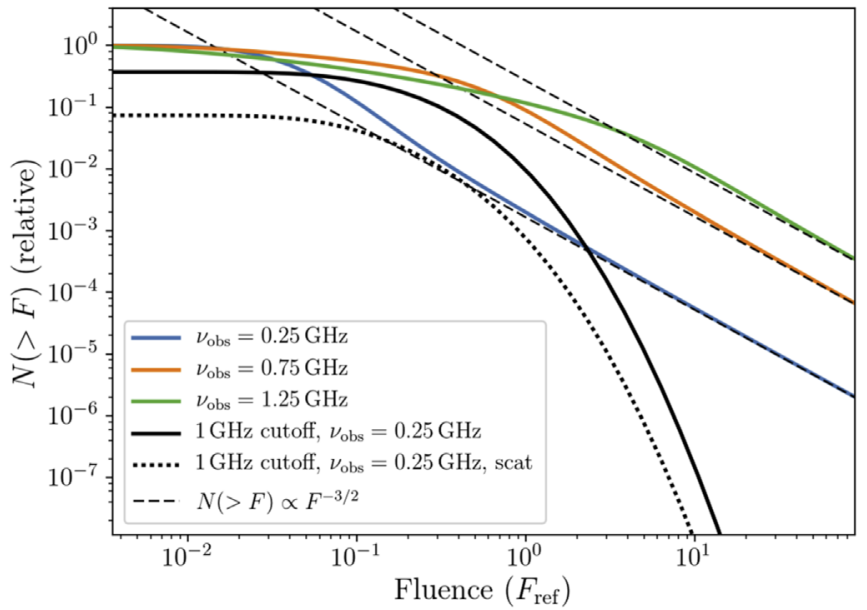

Figure 4. Observed integral FRB fluence distributions (Equation (14)) for $\nu_{\text {peak }}=2 \mathrm{GHz}$ at $\nu_{\text {obs }}=0.25,0.75,1.25 \mathrm{GHz}$ and a $[\alpha=-1.8, \beta=2.1]$ FRB spectrum (blue, orange, and green curves), and for $\nu_{\text {peak }}=1 \mathrm{GHz}$ and $\nu_{\text {obs }}=0.25 \mathrm{GHz}$ for the sharply cutoff spectrum considered above (black solid curve). The black dotted curve shows the latter case when the effects of temporal broadening, as specified above $\left(f_{b, \text { ref }}=0.1\right)$, are included. The assumptions regarding the FRB luminosity function are the same as above (e.g., Figures 2 and 3). The dashed black lines show indicative fluence distributions corresponding to uniformly distributed sources in Euclidean space $\left(N(>F) \propto F^{-3 / 2}\right)$.

is evident when the temporal-broadening scenario considered here is incorporated, albeit with a lower overall detection rate. In the case of a sharp spectral cutoff, sensitive low-frequency observations will predominantly detect high-redshift events (see also Fialkov \& Loeb 2017).

\section{Discussion}

\subsection{Postdictions}

We have demonstrated the possible effects of a characteristic low-frequency spectral cutoff for FRBs on some of the observed population statistics. The precise nature of the frequency-dependent rate, and the redshift and fluence distributions, depend sensitively on the FRB luminosity function, and the form of the characteristic rest-frame spectrum. 
This is the case even in the absence of cosmic evolution in the population. Furthermore, the frequency of the spectral cutoff, although plausibly at $\mathrm{GHz}$ frequencies, itself depends sensitively on the nature of FRB environments. These quantities are all poorly constrained at present. We therefore do not attempt to directly model the observed FRB population in this paper.

However, it is clear that a low-frequency spectral cutoff can explain the tight constraints on the characteristic FRB spectral index between the Parkes/ASKAP frequency bands, and the GBT $350 \mathrm{MHz}$ (Chawla et al. 2017) and MWA 170-200 MHz surveys (Sokolowski et al. 2018). For example, the results shown in Figure 2 demonstrate that equivalent detection rates for surveys with identical fluence thresholds can be obtained at $350 \mathrm{MHz}$ and $1.4 \mathrm{GHz}$ for a rest-frame turnover frequency $\nu_{\text {peak }} \sim 1 \mathrm{GHz}$. The general requirement for a frequencydependent FRB rate, with a maximal-rate frequency that is correlated with $\nu_{\text {peak }}$, is that the FRB luminosity function has a form such that brighter FRBs are generally observed at lower luminosity distances. This can be achieved in several ways besides with the log-normal form for the luminosity function we assume above, such as with a very steeply negative or cutoff power law. This may indeed be the case in reality if FRB DMs are a proxy for cosmological distance (Shannon et al. 2018).

A characteristic low-frequency spectral cutoff can also account for the differences between the Parkes and ASKAP FRB samples. The Parkes sample appears to have a burst fluence distribution that is flatter than the Euclidean-space expectation (approximately $\propto F^{-1}$; Vedantham et al. 2016; James et al. 2018), whereas the ASKAP sample appears to have a much steeper fluence distribution (approximately $\propto F^{-2.2}$; James et al. 2018). ${ }^{7}$ The ASKAP detection rate is also lower than expected from an extrapolation of the Parkes fluence distribution. A model for the FRB fluence distribution wherein a break exists between the Parkes and ASKAP fluencedetection regimes is thus favored (see also Amiri et al. 2017). This was attributed by Shannon et al. (2018) and James et al. (2018) to a cosmologically evolving population, with a higher volumetric rate at higher redshifts. However, a similar FRB fluence distribution, containing portions that are both flatter and steeper than the fiducial $F^{-3 / 2}$ law, can also be generated by observations at low frequencies relative to a rest-frame cutoff (e.g., Figure $4, \nu_{\text {obs }}=0.25 \mathrm{GHz}$ curves in both panels). In this model, the higher-fluence ASKAP events are observed typically from lower redshifts than the Parkes events (consistent with their lower DMs; Shannon et al. 2018), and the Parkes rate at higher redshifts is boosted by a negative $K$ correction. We stress that the analysis in Section 3 is not intended to present a quantitative prediction of the cutoff frequency $\nu_{\text {peak }}$ in this scenario, although it is likely to be $\gtrsim 2 \mathrm{GHz}$. We also remark that the differing spectral properties of the Parkes and ASKAP FRBs (Macquart et al. 2019) may also result from different regions of the characteristic rest-frame FRB spectrum being observed by the two surveys.

The steeply negative spectral index of $\alpha=-1.6_{-0.2}^{+0.3}$ between 1129 and $1465 \mathrm{MHz}$ measured by Macquart et al.

\footnotetext{
There has been substantial uncertainty regarding the Parkes FRB fluence distribution (e.g., Oppermann et al. 2016; Vedantham et al. 2016; Macquart \& Ekers 2018a). The continued detections of Parkes FRBs in multiple beams of the 13-beam array (e.g., Oslowski et al. 2018), together with the reassessment of the Macquart \& Ekers (2018a) technique by James et al. (2018), points toward a growing consensus in favor of a flat fluence distribution among Parkes FRBs.
}

(2019) for the ASKAP FRB sample presents a challenge to our proposed scenario. Given this observation, it is difficult to simultaneously explain the GBT/MWA nondetections and the differing properties of the Parkes and ASKAP samples using a single characteristic low-frequency cutoff. It is possible that ASKAP does indeed observe FRBs at rest frequencies $\nu>\nu_{\text {peak }}$, in which case cosmic evolution may be required to explain the differing source counts of the Parkes and ASKAP samples. Alternatively, it is possible that this measurement is erroneous, in which case the tension with a concordance scenario of a single $\nu_{\text {peak }}$ is removed. For example, accurate estimates of the FRB rest-frame spectrum may rely on redshift corrections being applied, which would be the case if some FRBs are observed near $\nu_{\text {peak }}$ and hence do not exhibit power-law spectra (as assumed by Macquart et al. 2019).

A further challenge to our hypothesis of a characteristic restframe spectral cutoff is the possibility that FRBs are viewed along a wide variety of sightlines. For example, FRB 110523 was potentially Faraday-rotated by magnetic fields slightly in excess of the typical Milky Way ISM, but was also more strongly scattered by the circum-burst medium (Masui et al. 2015). FRB 150807, on the other hand, appeared to be neither scattered nor Faraday-rotated by a potential host-galaxy ISM comparable to that of the Milky Way (Ravi et al. 2016). Although the repeating FRB 121102 may not share a progenitor with the remainder of the population (e.g., Shannon et al. 2018; Ravi 2019), its environment appears significantly different again, hosting $\gtrsim \mathrm{mG}$ magnetic fields but potentially not strongly scattering the bursts (e.g., Michilli et al. 2018). A variety of host environments may result in a range of values of

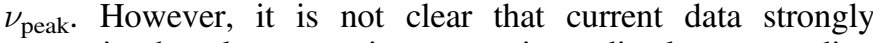
constrain the plasma environments immediately surrounding FRB sources on subparsec scales, because of the low column densities (of order unity) involved, and the suppression of scattering owing to the extreme geometry. Both the stimulated Raman and induced Compton scattering mechanisms only require significant plasma densities rather than column densities, and Razin-Tsytovich suppression only requires thermal plasma in the vicinity of the emission region. The existence of a characteristic rest-frame low-frequency cutoff therefore may rely on there being a common FRB progenitor, rather than a common progenitor environment.

\subsection{Predictions and Future Measurements}

The presence of a characteristic low-frequency spectral cutoff for FRBs has specific predictions that enable this scenario to be tested. These tests can also lead to a measurement of the cutoff frequency, $\nu_{\text {peak }}$, given some knowledge or assumptions about the form of the spectrum, and the FRB luminosity function. Such tests are important, because the observations discussed above can have other explanations. For example, even if the ASKAP spectral-index measurement (Macquart et al. 2019) is in error, an alternative explanation for the GBT and MWA nondetections is a characteristically flat FRB spectrum, such as that inferred for the repeating FRB 121102 between 1 and $8 \mathrm{GHz}$ (Gajjar et al. 2018).

Once a large FRB sample with redshift measurements becomes available, the mean rest-frame spectrum can be estimated by stacking redshift-corrected FRB spectra. If the difference between the Parkes and ASKAP FRB samples can 
be explained by a negative $K$-correction boosting the Parkes FRB rate at higher redshifts, the FRBs typically detected around $1.4 \mathrm{GHz}$ need to be observed below or near the restframe spectral peaks. This would also be consistent with the GBT/MWA nondetections at low frequencies. The required large FRB samples will be provided by localization observations with ASKAP, and by the Deep Synoptic Array; we note that bandpass calibration of these observations even at the offboresight beam locations will be critical. These measurements will also enable the FRB luminosity function to be estimated, which needs to have a form such that fainter events are typically more cosmologically distant.

Prior to the assembly of a large sample of FRBs with redshifts, insight into the existence of a low-frequency spectral cutoff will be provided by an analysis of the detection rate at different frequencies. A unique prediction of our hypothesis is the existence of a specific observing frequency with a maximum FRB detection rate for a fixed fluence threshold, although the effects of temporal broadening can mitigate the significance of this maximum. As shown in Figure 2, this will typically occur at a frequency somewhat below the rest-frame $\nu_{\text {peak }}$, when the negative $K$-correction maximizes the detection volume. If the negative observed spectral index estimated by Macquart et al. (2019) is a true indicator of the rest-frame spectral indices of the ASKAP sample, the GBT and MWA constraints would imply that the FRB detection rate may peak around the CHIME frequency band. On the other hand, if ASKAP is instead observing FRBs below or near their restframe $\nu_{\text {peak }}$, the FRB rate may peak at frequencies above $1.4 \mathrm{GHz}$.

We have also shown that a characteristic low-frequency spectral cutoff for FRBs will result in substantively different redshift and fluence distributions for different observing frequencies. Observations at low frequencies, below the frequency with the peak FRB rate, will preferentially detect more distant FRBs than observations at higher frequencies (Figure 3). This can result in low-frequency observations revealing FRB samples with relatively flat fluence distributions close to their detection thresholds (Figure 4). Observations at higher frequencies will preferentially detect events at lower redshifts, revealing steeper or Euclidean-space fluence distributions. The magnitudes of these effects will depend on the steepness of the rest-frame FRB spectrum both below and above $\nu_{\text {peak }}$.

CHIME, with its large $400-800 \mathrm{MHz}$ band and expected detection rate of a few FRBs per day when operational (The CHIME/FRB Collaboration et al. 2018), will likely have the greatest impact in the coming few years on testing the predictions of a characteristic low-frequency spectral cutoff for FRBs. The currently published results are subject to too many unknown observational effects, in particular due to the unknown observing efficiency and bandpass calibration, to reach even indicative conjectures (CHIME/FRB Collaboration et al. 2019). In the future, it will be important to use CHIME to conduct searches in subsections of the observing band, with known completeness thresholds in fluence, DM, and temporal width. This will enable the frequency with the maximum FRB rate to be inferred, and comparisons to be made between the DM and fluence distributions of FRBs observed at different frequencies.

\section{Conclusions}

We have examined the possibility of a low-frequency cutoff in the characteristic rest-frame spectrum of FRBs. We conclude the following:

1. A selection of effects can result in the absorption or suppression of FRB emission at low frequencies, regardless of the specific emission mechanism (Figure 1). The extreme brightness temperatures of FRBs means that induced Compton scattering will occur for all reasonable thermal-plasma progenitor environments. Stimulated Raman-scattering interactions with Langmuir waves and Razin-Tsytovich suppression will also occur for dense plasma (e.g., $n_{e} \sim 10^{6} \mathrm{~cm}^{-3}$ for $T_{e} \sim 10^{6} \mathrm{~K}$ ). Free-free absorption may be relevant in particularly dense, cold environments. Examples of low-frequency spectral cutoffs exist in practice among the Galactic pulsar population. The potentially complex effects of the $K$-correction, beyond the power-law models previously considered (Vedantham et al. 2016; Macquart \& Ekers 2018 b), must therefore be incorporated into predictions of cosmological FRB population models (see Fialkov \& Loeb 2017).

2. A characteristic low-frequency spectral cutoff for FRBs will, under a variety of assumptions, manifest in the FRB rate being maximized for a particular observing frequency (Figure 2). Relative to higher frequency observations, surveys below the maximal-rate frequency will preferentially detect higher-redshift events (Figure 3), and will result in samples with sharply broken fluence distributions (Figure 4). High-frequency observations will be more likely to detect nearby events. These results are more pronounced for sharper spectral cutoffs, with observations below the maximal-rate frequency yielding more faint high-redshift events, and fewer bright nearby events.

3. We suggest that the differences between the Parkes and ASKAP FRB samples, together with the nondetections of FRBs at low frequencies, can be explained by the suppression of low-frequency FRB emission even if the population does not evolve with cosmic time. A difficulty with this scenario is the steeply negative spectral index that may be characteristic of ASKAP FRBs (Macquart et al. 2019). Our hypothesis will be tested by measurements of the FRB rate and fluence distribution at multiple frequencies, in particular by CHIME. If FRB DMs form a good proxy for cosmological redshift, the FRB DM distributions at different frequencies will also be revealing. Direct measurements of the characteristic FRB restframe spectrum with FRBs localized to host galaxies with redshift measurements will provide a further test.

V.R. thanks R. Blandford for raising the possibility of stimulated Raman scattering in FRB plasma environments. This work was supported in part by grants from the Breakthrough Prize Foundation and the black hole Initiative through the John Templeton Foundation.

\section{ORCID iDs}

Vikram Ravi (iD https://orcid.org/0000-0002-7252-5485

Abraham Loeb (1) https://orcid.org/0000-0003-4330-287X 


\section{References}

Akhiezer, A. I., Akhiezer, I. A., Polovin, R. V., Sitenko, A. G., \& Stepanov, K. N. 1975, Plasma Electrodynamics. Volume 1-Linear Theory. Volume 2-Nonlinear Theory and Fluctuations (Oxford: Pergamon)

Alexander, K. D., Berger, E., Guillochon, J., Zauderer, B. A., \& Williams, P. K. G. 2016, ApJL, 819, L25

Amiri, M., Bandura, K., Berger, P., et al. 2017, ApJ, 844, 161

Arons, J., \& Barnard, J. J. 1986, ApJ, 302, 120

Bailes, M., Jameson, A., Flynn, C., et al. 2017, PASA, 34, e045

Bilous, A. V., Kondratiev, V. I., Kramer, M., et al. 2016, A\&A, 591, A134

Boischot, A., \& Clavelier, B. 1967, ApL, 1, 7

Bondi, H. 1952, MNRAS, 112, 195

Boyle, P. C. \& Chime/Frb Collaboration 2018, ATel, 11901

Burke-Spolaor, S., \& Bannister, K. W. 2014, ApJ, 792, 19

Burke-Spolaor, S., Trott, C. M., Brisken, W. F., et al. 2016, ApJ, 826, 223

Caleb, M., Flynn, C., Bailes, M., et al. 2017, MNRAS, 468, 3746

Chawla, P., Kaspi, V. M., Josephy, A., et al. 2017, ApJ, 844, 14

CHIME/FRB Collaboration, Amiri, M., et al. 2019, Natur, 566, 230

Coppi, P., Blandford, R. D., \& Rees, M. J. 1993, MNRAS, 262, 603

Cordes, J. M., \& Wasserman, I. 2016, MNRAS, 457, 232

Cordes, J. M., Wasserman, I., Hessels, J. W. T., et al. 2017, ApJ, 842, 35

Deneva, J. S., Stovall, K., McLaughlin, M. A., et al. 2016, ApJ, 821, 10

Draine, B. T. (ed.) 2011, Physics of the Interstellar and Intergalactic Medium (Princeton, NJ: Princeton Univ. Press)

Fialkov, A., \& Loeb, A. 2017, ApJL, 846, L27

Gaensler, B. M., \& Slane, P. O. 2006, ARA\&A, 44, 17

Gajjar, V., Siemion, A. P. V., Price, D. C., et al. 2018, ApJ, 863, 2

Gangadhara, R. T., \& Krishan, V. 1992, MNRAS, 256, 111

Ginzburg, V. L., \& Syrovatskii, S. I. 1965, ARA\&A, 3, 297

Hankins, T. H., \& Eilek, J. A. 2007, ApJ, 670, 693

Ho, A. Y. Q., Phinney, E. S., Ravi, V., et al. 2019, ApJ, 871, 73

Hogg, D. W., Baldry, I. K., Blanton, M. R., \& Eisenstein, D. J. 2002, arXiv:astro-ph/0210394

Ioka, K. 2003, ApJL, 598, L79

James, C. W., Ekers, R. D., Macquart, J.-P., Bannister, K. W., \& Shannon, R. M. 2018, MNRAS, 483, 134

Jankowski, F., van Straten, W., Keane, E. F., et al. 2018, MNRAS, 473, 4436

Karastergiou, A., Chennamangalam, J., Armour, W., et al. 2015, MNRAS, 452, 1254

Katz, J. I. 2014, PhRvD, 89, 103009

Keane, E. F., \& Petroff, E. 2015, MNRAS, 447, 2852

Kijak, J., Lewandowski, W., Maron, O., Gupta, Y., \& Jessner, A. 2011, A\&A, 531, A16
Kramer, M., Karastergiou, A., Gupta, Y., et al. 2003, A\&A, 407, 655

Kulkarni, S. R., Ofek, E. O., \& Neill, J. D. 2015, arXiv:1511.09137

Kumar, P., Lu, W., \& Bhattacharya, M. 2017, MNRAS, 468, 2726

Law, C. J., Abruzzo, M. W., Bassa, C. G., et al. 2017, ApJ, 850, 76

Levinson, A., \& Blandford, R. 1995, MNRAS, 274, 717

Lyubarsky, Y. 2008, ApJ, 682, 1443

Lyubarsky, Y., \& Ostrovska, S. 2016, ApJ, 818, 74

Macquart, J.-P., \& Ekers, R. D. 2018a, MNRAS, 474, 1900

Macquart, J.-P., \& Ekers, R. D. 2018b, MNRAS, 480, 4211

Macquart, J.-P., \& Koay, J. Y. 2013, ApJ, 776, 125

Macquart, J.-P., Shannon, R. M., Bannister, K. W., et al. 2019, ApJ, 872 , L19

Margalit, B., Metzger, B. D., Berger, E., et al. 2018, MNRAS, 481, 2407

Masui, K., Lin, H.-H., Sievers, J., et al. 2015, Natur, 528, 523

Melrose, D. B. 1972, Ap\&SS, 18, 267

Metzger, B. D., Berger, E., \& Margalit, B. 2017, ApJ, 841, 14

Meyers, B. W., Tremblay, S. E., Bhat, N. D. R., et al. 2017, ApJ, 851, 20

Michilli, D., Seymour, A., Hessels, J. W. T., et al. 2018, Natur, 553, 182

Mickaliger, M. B., McEwen, A. E., McLaughlin, M. A., \& Lorimer, D. R. 2018, MNRAS, 479, 5413

Mikami, R., Asano, K., Tanaka, S. J., et al. 2016, ApJ, 832, 212

Murphy, T., Kaplan, D. L., Bell, M. E., et al. 2017, PASA, 34, e020

Newburgh, L. B., Bandura, K., Bucher, M. A., et al. 2016, Proc. SPIE, 9906, 99065X

Oppermann, N., Connor, L. D., \& Pen, U.-L. 2016, MNRAS, 461, 984

Oslowski, S., Shannon, R. M., Jameson, A., et al. 2018, ATel, 11385

Planck Collaboration, Ade, P. A. R., Aghanim, N., et al. 2016, A\&A, 594, A13

Platts, E., Weltman, A., Walters, A., et al. 2018, arXiv:1810.05836

Rajwade, K., Lorimer, D. R., \& Anderson, L. D. 2016, MNRAS, 455, 493

Rajwade, K. M., \& Lorimer, D. R. 2017, MNRAS, 465, 2286

Ravi, V. 2018, ApJ, 872, 88

Ravi, V. 2019, MNRAS, 482, 1966

Ravi, V., Shannon, R. M., Bailes, M., et al. 2016, Sci, 354, 1249

Rowlinson, A., Bell, M. E., Murphy, T., et al. 2016, MNRAS, 458, 3506

Shannon, R. M., Macquart, J.-P., Bannister, K., et al. 2018, Natur, 562, 386

Sokolowski, M., Bhat, N. D. R., Macquart, J. P., et al. 2018, ApJ, 867, L12

Sunyaev, R. A. 1971, SvA, 15, 190

The CHIME/FRB Collaboration, Amiri, M., Bandura, K., et al. 2018, ApJ, 863,48

Tendulkar, S. P., Bassa, C. G., Cordes, J. M., et al. 2017, ApJL, 834, L7

Vedantham, H. K., \& Phinney, E. S. 2019, MNRAS, 483, 971

Vedantham, H. K., Ravi, V., Hallinan, G., \& Shannon, R. M. 2016, ApJ, 830,75

Wilson, D. B., \& Rees, M. J. 1978, MNRAS, 185, 297

Yang, Y.-P., Zhang, B., \& Dai, Z.-G. 2016, ApJL, 819, L12 